\title{
«On ne cherche pas des gens préparés à être ministre mais des gens qui sont prêts au changement »
}

Entretien avec Joséphine Ouédraogo, ancienne ministre de l'Essor familial et de la Solidarité nationale (1984-1987) lors de la présidence de Thomas Sankara

\section{Partie 2}

\author{
Hadrien Clouet et Maxime Quijoux
}

\begin{abstract}
Citer cet article : Clouet Hadrien, Quijoux Maxime (2021), " "On ne cherche pas des gens préparés à être ministre mais des gens qui sont prêts au changement". Entretien avec Joséphine Ouédraogo (Partie 2) », Revue d'Histoire Contemporaine de l'Afrique, en ligne. URL :

https://oap.unige.ch/journals/rhca/article/view/entclouetquijoux2

Mise en ligne : 14 juin 2021

DOI : https://doi.org/10.51185/journals/rhca.2021.e498
\end{abstract}

Suite de l'entretien de Joséphine Ouédraogo par Hadrien Clouet et Maxime Quijoux. Pour retrouver la première partie : https://oap.unige.ch/journals/rhca/article/view/entclouetquijoux

\section{Les luttes de l'exil}

\section{Hadrien Clouet : Comment avez-vous vécu la fin du processus révolutionnaire et l'assassinat du président Sankara le 15 octobre 1987 ?}

C'était un choc brutal pour moi comme pour tout le monde. J'étais en mission hors du pays au moment du coup d'État... Thomas Sankara a accepté que j'aille à Genève, sur l'invitation d'une grande ONG, pour prendre la parole à l'occasion de la Journée mondiale de l'alimentation, célébrée le 16 octobre de chaque année. II m'a dit : « II faut y aller, surtout pour la journée de I'alimentation, tu dois donner le message que nous ne voulons plus être nourris sous perfusion, mais que nous voulons produire ce dont nous avons besoin, nous voulons résoudre le problème de la sécurité alimentaire par la souveraineté nationale ». Bref, j'avais un message à donner. Je lui ai dit au revoir avant de partir.

Le 15 octobre à 20 heures, j'ai été alertée par un ami journaliste du coup d'État et j'ai entendu la confirmation officielle en écoutant le journal télévisé dans ma chambre d'hôtel. Avant mon départ de Ouaga, le climat politique était très tendu et nous étions conscients qu'il pourrait y avoir un coup d'État, jusqu'à son accomplissement sanglant le 15 octobre qui a coûté la vie au président Thomas Sankara et douze de ses compagnons politiques. J'étais hors du 
pays et j'avais le choix : rester à l'extérieur ou rentrer. Pour moi, il n'était pas question de rester dehors, parce que cela laissait entendre que je me sentais coupable de quoi que ce soit. J'étais suffisamment engagée dans la vision sociale révolutionnaire pour devoir en assumer les conséquences, non sans une certaine peur. Dès que les frontières furent ouvertes, j'ai donc signalé ma position à tout le monde, en signifiant que je ne suis pas en fuite et que je rentrerai par le premier vol disponible.

À mon retour, j'ai constaté qu'en plus de la mort de Thomas Sankara et de douze collègues que je connaissais, certains de mes camarades ministres étaient emprisonnés. En arrivant, j'ai été assignée à résidence pendant quelques semaines. Mon mari était en poste en Tunisie depuis quelques mois. II y était avec les enfants. Au mois de décembre 1987, mon assignation à résidence étant levée, j'ai demandé une audience avec le président Blaise Compaoré pour lui demander l'autorisation de rejoindre ma famille à Tunis. Il a accepté et j'ai quitté le Burkina jusqu'en juillet 1992. Rentrée pour ouvrir mon propre bureau d'études, je suis repartie en 1997 à la CEA [Commission économique pour l'Afrique des Nations Unies], puis à Enda-Tiers-monde jusqu'en 2012.

\section{Durant ces années d'exil, vous avez pu continuer vos luttes politiques d'une autre façon, en conduisant des projets progressistes dans les institutions internationales?}

Quand j'étais à la CEA de 1997 à 2007 comme directrice du Centre africain genre et développement [CAGED] au sein de la Commission économique des Nations Unies pour I'Afrique basée à Addis-Abeba, notre mandat était d'influencer les politiques africaines de promotion de l'égalité des genres et de promotion des femmes, notamment sur le champ économique. Le secrétaire exécutif de la CEA de l'époque, quand il m'a recrutée, a dit ceci : " Je vous ai sélectionnée car vous n'êtes pas une académicienne féministe. Je vois que vous avez été impliquée dans les questions de développement et que vous avez une expérience gouvernementale. Nous devons contribuer à changer les politiques nationales en faveur des femmes ».

Deux actions ont marqué mon expérience professionnelle à la CEA. Premièrement, le secrétaire exécutif a décidé de célébrer le $40^{\mathrm{ème}}$ anniversaire de la création de l'organisation par une conférence internationale sur le thème de l'égalité hommes/femmes. Le public prioritaire de cette conférence était composé des ministres des Finances ou de l'Économie, principaux membres de l'organe décisionnel de la CEA. Ils devaient être aux premières loges de la célébration du $40^{\text {ème }}$ anniversaire de l'organisation. Pour imprimer une composition plurielle des participants à la conférence, j'ai proposé d'inviter les ministres africains chargés de l'Agriculture, de la Santé, de la Recherche, de l'Éducation, de l'Action sociale et du Travail. Le format de la conférence prévoyait une série d'ateliers thématiques portant sur la prise en compte de l'approche genre et de la promotion des femmes dans les politiques sectorielles. La conférence a réuni près de 2000 participants issus du secteur public, privé et de la société civile, autour du thème de l'égalité hommes/femmes.

Deuxièmement, comme je l'ai mentionné plus haut, la mission du CAGED était de dresser tous les cinq ans, en collaboration avec les gouvernements, le bilan de la mise en œuvre de la 
Plateforme d'action de Beijing à l'échelle du continent africain : Beijing +5 , Beijing $+10^{1} \ldots$ Mais dans les faits, au cours de ces bilans périodiques, chaque pays venait exposer ses success stories, ses difficultés, ses programmes prioritaires et ses besoins financiers. C'était une approche qui reposait sur l'auto-évaluation par les gouvernements des résultats de leurs propres politiques.

J'ai proposé à l'équipe du CAGED d'élaborer un instrument de mesure objective, pour ne pas dire scientifique, des inégalités des sexes dans plusieurs domaines. Avec l'implication de statisticiens, de chercheurs, d'experts en genre et la collaboration du FNUAP [Fonds des Nations Unies pour la population], de l'UNIFEM [Fonds de développement des Nations Unies pour la femme] et du PNUD [Programme des Nations Unies pour le développement], nous avons travaillé pendant un an pour développer et tester un indice de mesure des inégalités, à partir de 43 indicateurs. Cet instrument, appelé I'IDISA (Indicateur du développement et des inégalités entre les sexes en Afrique) a été testé et validé en 2005 dans une dizaine de pays africains. Une fois diffusé à l'échelle du continent, il devait permettre de mesurer et de comparer l'évolution des inégalités hommes/femmes à l'échelle du continent.

\section{$\underline{\text { L'insurrection de 2014, une légitimation populaire de Sankara }}$}

\section{Et vous renouez avec la politique burkinabè en $2014 \ldots$}

L'insurrection populaire de 2014 et la période de transition (2015) ont contribué à la légitimation populaire de la révolution sankariste. Les insurgés réclamaient l'abolition de la corruption, la lutte contre la pauvreté, le jugement des crimes politiques et économiques, la fin de l'assujettissement de la justice au régime en place, et le traitement des dossiers d'assassinats politiques bloqués depuis deux décennies.

J'ai été appelée au gouvernement de la transition comme ministre de la Justice. J'ai espéré et pensé que cette étape post-insurrectionnelle nous offrait l'opportunité de jeter les bases d'un processus de changement politique et social.

\section{Justement, un point qui nous intrigue, c'est que vous avez conduit cette transition au ministère de la Justice. À quoi est due la nomination à ce poste-là ?}

Après l'insurrection populaire et le départ du président Blaise Compaoré, forcé de s'exiler, la classe politique nationale appuyée par la communauté internationale a exigé la mise en place d'un gouvernement de transition présidé par un homme ou une femme, civil et reconnu, entre autres pour ses qualités d'intégrité, de patriotisme, de maturité et ayant le sens des responsabilités. Ils ont aligné des tas de vertus... je me suis demandé « où va-t-on trouver quelqu'un comme ça ? » [Rires].

La société civile, les partis politiques et des organisations diverses ont fait des propositions et mon nom faisait partie de la liste des femmes proposées comme éligibles pour

\footnotetext{
${ }^{1}$ La $4^{e}$ conférence mondiale des Nations Unies sur les femmes se tient à Beijing en 1995. Elle prévoit des évaluations quinquennales du plan d'action.
} 
la présidence de la transition. Un samedi matin, on m'explique au téléphone que le jury allait tenter de s'accorder sur l'un de nous. À défaut, il faudrait passer en audition. Le jury avait toute la journée pour trancher, jusqu'à 19 heures. À minuit, le téléphone sonne: ils n'ont pas su s'accorder, il faut venir en interview. Tour à tour, chacun de nous a été interrogé par ce jury : questions, réponses, questions, réponses... À la sortie, ils ont choisi monsieur Michel Kafando².

Le jury a probablement décidé d'attribuer des postes-clés aux deux candidats perdants. L'autre candidat [Moumina Chériff Sy], a obtenu la présidence du Conseil national de transition (Assemblée nationale), et on m'a proposé le ministère de la Justice. Mon engagement pour le changement m'a certainement poussé à accepter cette fonction malgré sa grande difficulté et les grands défis que représentaient les revendications des insurgés, parmi lesquels figurait l'indépendance de la justice.

\section{Et quelle a été votre stratégie politique à ce poste ?}

II fallait faire vite pour jeter les bases politiques et institutionnelles d'une indépendance de la justice : revisiter les textes, obtenir l'approbation des pouvoirs publics notamment l'exécutif, et d'abord convaincre les magistrats de la nécessité de leur propre engagement à se démarquer des faits qui leur sont reprochés par les insurgés ${ }^{3}$.

Je ne voyais qu'une seule démarche possible: organiser les États généraux de la Justice. Il s'agissait d'une rencontre nationale, au cours de laquelle nous devions mettre à plat tous les travers du système judiciaire et de ses acteurs, mais aussi les comportements et les influences de l'exécutif et de certaines catégories de justiciables qui se croient au-dessus des lois.

La rencontre a été préparée avec l'implication des acteurs du ministère de la Justice ainsi que les populations : celles-ci ont été conviées à s'exprimer à travers des forums préparatoires organisés dans cinq régions. Ces réflexions ont permis d'organiser les États généraux dans une démarche de concertation publique structurée autour d'ateliers thématiques. En effet, tout le système s'enfermait sur lui-même pour élaborer des lois. Pour le Code de la famille, dans les années 1980, on avait consulté les femmes en organisant des journées nationales, alors que les magistrats ne consultaient plus personne. Là, c'est aussi la formation de sociologue qui m'a sensibilisée à la connexion entre la société et les acteurs techniques et administratifs, entre la population et ceux qui dirigent. II y a certes de beaux textes de loi, que les parlementaires s'enferment pour corriger et adopter, tous en français. Que font les institutions dites démocratiques, pour les diffuser auprès des populations et créer des mécanismes d'explication au niveau local ?

Le principal résultat attendu était le Pacte national pour le renouveau de la Justice, une sorte de contrat social et politique qui présente les responsabilités de chaque partie dans la mise en œuvre d'un processus de réforme de la justice et d'instauration de son indépendance.

2500 participants ont travaillé pendant cinq jours. Ils étaient issus de toutes catégories socioprofessionnelles : membres de l'exécutif et du judiciaire, autres services publics, services

\footnotetext{
2 Qui disposait d'un argument de poids, à savoir le soutien de l'armée. Saidou Abdoul Karim (2017), « Burkina Faso : où en est la réforme de l'armée, deux ans après l'insurrection populaire ", Note d'analyse du GRIP, pp. 1-16.

3 Disparitions ou assassinats, procès politiques, procédures expéditives et arbitraires, etc.
} 
privés, organisations de la société civile, artisans, agriculteurs, éleveurs, organisations de femmes, etc. J'avais demandé aux magistrats de se contenter d'abord d'écouter toutes les critiques qui seraient formulées contre eux et le système judiciaire au cours de la première journée des États généraux qui était consacrée à l'état des lieux. Je leur ai dit ceci : « C'est l'opportunité unique qui vous est offerte pour vous réconcilier avec le peuple burkinabè après I'insurrection. À la limite, on peut dire que vous êtes ce que vous êtes faute d'indépendance, car l'exécutif vous tenait sous tutelle, mais désormais les gens vous veulent libres, c'est donc l'occasion de légitimer votre quête d'indépendance et de liberté ».

À l'issue des travaux, le Pacte a été adopté et signé par tous les protagonistes : le Premier ministre, la magistrature, les ONG, la société civile, et les partis politiques. À la fin de la période de transition, les magistrats ont obtenu leur indépendance par décret, et le Conseil supérieur de la magistrature n'est plus présidé par le président du Faso. Cependant, un tel Pacte qui regroupe une soixantaine d'articles couvrant les aspects essentiels sur lesquels doit s'opérer la réforme du système judiciaire et de ses acteurs, requiert au moins une décennie pour sa mise en œuvre, à condition qu'il y ait un effort soutenu et volontariste de la part des protagonistes.

\section{Deux fois ministre, à trente ans d'écart, quelles évolutions avez-vous ressenti ?}

En 1985, le Burkina (Haute-Volta à l'époque) faisait partie des pays les plus pauvres, si l'on se fie aux indicateurs de développement définis par la communauté internationale. Majoritairement analphabète, la population était en forte croissance mais n'atteignait en moyenne pas les 50 ans, tandis que les médecins demeuraient très rares.

La révolution conduite par Thomas Sankara voulait changer ce tableau à travers trois principaux leviers. Premièrement, imprimer une vision de progrès économique et social à l'ensemble de la population et tous les acteurs publics et privés intervenant dans le développement : I'objectif étant de susciter un élan de responsabilité collective pour mener le changement. Deuxièmement, reconsidérer la place et le rôle des institutions d'aide au développement (publiques, privées, ONG, bilatérales et multilatérales); elles ne devaient plus être les décideuses, les moteurs et les bailleurs du développement du Burkina. Elles devaient devenir des accompagnatrices du gouvernement et des populations dans un processus de progrès conduit par les dirigeants nationaux. Troisièmement, considérer que le processus du développement national est un tout ; tous les secteurs et tous les acteurs sont interdépendants. Privilégier un secteur par rapport à un autre engendre des déséquilibres économiques et des exclusions sociales.

Le processus révolutionnaire a duré seulement 4 ans. Après cela, le Burkina est entré dans le système international de développement économique et s'est aligné sur le mode de gouvernance démocratique initialement hérité de la période coloniale. Le Burkina s'est intégré à la mondialisation et applique les principes de gestion économique et financière imposés par les institutions internationales.

Désormais, le Burkina affiche un taux de croissance économique remarquable oscillant entre 5 et $7 \%$ (qui lui a valu les félicitations des institutions financières internationales) mais parallèlement le pays est classé parmi les 10 pays les plus pauvres de la planète selon l'indice de développement humain (IDH). Cette contradiction est révélatrice de l'écart qui n'a cessé de 
s'élargir entre les catégories sociales économiquement et politiquement aisées (qui profitent le plus de la croissance et des investissements internationaux) et la grande majorité de la population qui s'appauvrit.

\section{Et désormais, après votre poste de ministre de la Justice, vous occupez des fonctions diplomatiques?}

Un an après mon départ du gouvernement de la transition, à la faveur de la mise en place du nouveau gouvernement à la suite des élections de fin 2015, j'ai été nommée ambassadrice en Italie et représentante permanente du Burkina à la FAO [Organisation des Nations Unies pour I'alimentation et l'agriculture], au FIDA [Fonds international de développement agricole] et au PAM [Programme alimentaire mondial].

J'ai aussi la responsabilité de soutenir les Burkinabè de la diaspora vivant en Italie ${ }^{4}$. Par ce biais, je me retrouve en première ligne d'observation du phénomène migratoire, face aux réalités de la migration, des chemins de la mort et du caractère suicidaire des trajets qui les mènent vers les côtes italiennes.

\section{Maxime Quijoux : Avez-vous l'impression qu'aujourd'hui, au Burkina, la mémoire de Thomas Sankara est présente, contestée, célébrée ? Quels sont les legs de la révolution et de vos contributions à cette époque?}

La mémoire de Thomas Sankara et de la révolution est plus que jamais vivante depuis 2014 . Durant l'insurrection populaire, les images de Thomas Sankara étaient affichées et brandies partout.

Auparavant, les personnes et les groupes qui se réclamaient de Thomas Sankara ou qui en faisaient la publicité pouvaient subir une certaine forme de répression ou d'exclusion. Le président Roch Marc Christian Kaboré a posé la première pierre du mémorial consacré à Thomas Sankara, en présence de personnalités internationales. Ce bâtiment va abriter les actes de la révolution et la pensée de Thomas Sankara, afin de nourrir la jeunesse burkinabè.

Récemment, le chef de l'État a également décidé de restaurer l'Institut des peuples noirs, créé sous Thomas Sankara et dont les activités ont été gelées après son assassinat. La vision de Thomas Sankara et son engagement ne portaient pas uniquement sur le sort du peuple burkinabè. II entretenait une vision planétaire de la justice sociale, de l'émancipation des peuples exclus et opprimés à cause de leurs origines, leurs races, leurs positions sociales. Les Noirs américains, la diaspora afro-descendante, voyaient enfin en lui la reconnaissance de leur propre dignité bafouée parce qu'ils ont été esclaves et descendants d'esclaves. La réhabilitation de Thomas Sankara serait incomplète sans la réhabilitation de I'Institut des peuples noirs.

\footnotetext{
${ }^{4}$ De 2007 à 2013, le nombre de Burkinabè immigrés en Italie a doublé, de 5200 à 11500 entrées annuelles, dont les trois-quarts pour un emploi salarié ou raisons familiales. OIM (2016), Étude sur la diaspora burkinabè au Burkina Faso, en Côte d'Ivoire, en Italie et en France, Ouagadougou, Représentation au Burkina Faso, p. 32.
} 


\section{Parcours de Joséphine Ouédraogo}

- 1949 : naissance en 1949 à Koudougou (Haute-Volta)

- 1968 : baccalauréat à Paris (France)

- 1971 : diplômée de l'Institut de service social et de recherches sociales de Montrouge (France).

- 1974 : licence de sociologie à Paris V - René Descartes (France).

- 1974: chargée d'études sociologiques à la Société africaine d'études et de développement SA (Haute-Volta).

- 1980 : chargée d'études sociologiques à l'Autorité des aménagements des vallées des Volta (Haute-Volta).

- 1984 : ministre de l'Essor familial et de la Solidarité nationale (Burkina Faso).

- 1987 : Séjour à l'extérieur du Burkina. Consultante et coordinatrice de projets à I'Institut panafricain pour le développement à Douala (Cameroun).

- 1992 : chargée d'études et de la formation à la SACED à Bussigny (Suisse).

- 1993 : fondatrice et directrice d'ARC, bureau d'études S.A.R.L. à Ouagadougou (Burkina Faso).

- 1995 : directrice générale de la Coopération internationale au ministère des Affaires étrangères (Burkina Faso).

- 1997 : directrice du Centre africain pour le genre et le développement (CAGED) de la Commission économique des Nations Unies pour l'Afrique (CEA) à Addis-Abeba (Éthiopie).

- 2005 : secrétaire exécutive adjointe ad interim de la Commission économique des Nations Unies pour l'Afrique (CEA)à Addis-Abeba (Éthiopie).

- 2007 : secrétaire exécutive de l'ONG Enda-Tiers Monde à Dakar (Sénégal).

- 2013 : directrice d'ARC, bureau d'études à Ouagadougou (Burkina Faso).

- 2014-2015 : ministre de la Justice, des Droits humains et de la Promotion civique, Garde des Sceaux sous la transition (Burkina Faso).

- 2016 : ambassadrice du Burkina Faso en Italie et auprès des agences onusiennes de la FAO, du PAM et de la FIDAO basées à Rome (Italie).

Hadrien Clouet
CERTOP, Toulouse (France)
Maxime Quijoux
LISE, Centre National des Arts et Métiers (France)

\section{Bibliographie}

OIM (2016), Étude sur la diaspora burkinabè au Burkina Faso, en Côte d'Ivoire, en Italie et en France, Ouagadougou, Représentation au Burkina Faso.

SAIDou Abdoul Karim (2017), «Burkina Faso: où en est la réforme de l'armée, deux ans après I'insurrection populaire », Note d'analyse du GRIP, pp. 1-16. 